\title{
Should Ventilator-Associated Events become a Quality Indicator for ICUs?
}

\author{
Michael Klompas MD MPH and Lorenzo Berra MD
}

\author{
Introduction \\ The Pro Position \\ Limitations of VAP as a Quality Metric for Ventilated Patients \\ The Genesis of VAE Definitions \\ Data Supporting the Potential Value of VAE as a Quality Indicator \\ Clinical Importance \\ Surveillance Efficiency \\ Capacity to Guide Improvements in Care (Preventability) \\ VAE Surveillance: A Patient Safety Opportunity \\ Concerns About the Potential Value of VAEs as a Quality Indicator \\ The Positive Predictive Value of VAEs for Pneumonia Is Low \\ The Sensitivity of VAE Definitions for VAP Is Low \\ VAE Surveillance Is Susceptible to Gaming \\ Most VAEs Are Not Preventable \\ The Con Position \\ The VAE Algorithm Is Confusing \\ VAE Surveillance Is Insensitive for Identifying VAP \\ VAE Surveillance Is Not a Comprehensive \\ Assessment of a Physiological System \\ VAE Surveillance Is Susceptible to Abuse and Misuse \\ VAEs Can Sometimes Be Triggered by Improvements in Care \\ Con Position Concluding Remarks \\ Conclusions
}

The United States Centers for Disease Control and Prevention replaced their longstanding ventilator-associated pneumonia (VAP) definitions with ventilator-associated event (VAE) definitions in 2013. Controversy abounds as to whether VAE definitions are potentially suitable to serve as quality indicators for ICUs. On the pro side, VAE definitions overcome many of the weaknesses of traditional VAP surveillance. VAE definitions are objective, reproducible, electronically computable, and strongly predict poor outcomes for patients. There is an increasing amount of data on clinical correlates, risk factors, and approaches to prevent VAEs. Potential strategies to prevent VAEs are highly aligned with accepted best practices in critical care. VAE surveillance therefore has the potential to catalyze better care and to help hospitals track outcomes in ventilated patients more rigorously and more efficiently. On the con side, the complete VAE definition set with subtiers is complicated, neither sensitive nor specific for VAP, non-physiological compared with other ICU metrics, susceptible to gaming, and may bring about changes in clinician behavior that could paradoxically end up harming patients. Key words: ventilator-associated events; ventilator-associated pneumonia; quality improvement; surveillance and prevention. [Respir Care 2016;61(6):723-736. ( 2016 Daedalus Enterprises] 


\section{VAEs AS A QUALITy INDICATOR IN THE ICU}

\section{Introduction}

The United States Centers for Disease Control and Prevention introduced ventilator-associated event (VAE) definitions in early 2013 as a potential new quality metric for ventilated patients. ${ }^{1}$ The new definitions were designed to overcome many of the shortcomings of traditional ventilator-associated pneumonia (VAP) definitions, including their complexity, subjectivity, and low attributable mortality. There is an accumulating body of evidence documenting the potential advantages of VAE over VAP surveillance, including their high attributable mortality, their suitability for automated surveillance, and their capacity to catalyze and reflect the adoption of best practices for ventilated patients. At the same time, however, critics have raised multiple concerns about VAEs, including their poor overlap with VAP, their susceptibility to gaming, and doubts about the extent to which they are preventable. In this article, we will explore the merits and limitations of VAE as a potential quality indicator for ventilated patients.

\section{The Pro Position}

\section{Limitations of VAP as a Quality Metric for Ventilated Patients}

VAP has long been the de facto means for hospitals and regulators to track quality of care for ventilated patients. Advocates point to VAP's frequency, morbidity, and high cost to support its use as a quality marker and argue that eliminating VAP would save tens of thousands of lives per year. ${ }^{2}$ In practice, however, VAP has proven to be a poor means to measure and drive quality improvement for ventilated patients. Weaknesses of VAP as a quality metric are summarized in Table 1. The fundamental problem is that

Dr Klompas is affiliated with the Department of Population Medicine, Harvard Medical School and Harvard Pilgrim Health Care Institute, and the Department of Medicine, Brigham and Women's Hospital, Boston, Boston, Massachusetts. Dr Berra is affiliated with the Department of Anesthesia, Massachusetts General Hospital, Boston, Massachusetts.

Drs Klompas and Berra presented a version of this paper at the 54th RESPIRATORY CARE Journal Conference, "Respiratory Care Controversies III," held June 5-6, 2015, in St Petersburg, Florida.

Dr Klompas discloses relationships with the Centers for Disease Control and Prevention and the Hospital Association of New York State. Dr. Berra has no conflicts to disclose.

Correspondence: Michael Klompas MD MPH, Department of Population Medicine, Harvard Medical School and Harvard Pilgrim Health Care Institute, 401 Park Drive, Suite 401, Boston, MA 02215. E-mail: mklompas@partners.org.

DOI: $10.4187 /$ respcare 04548
VAP diagnosis is subjective and nonspecific. Typical diagnostic criteria include signs such as "new or progressive infiltrates," "increased secretions," and "worsening oxygenation." These signs are all subjective; hence, reasonable people are prone to disagree. Indeed, a wealth of studies attests to high rates of disagreement between different surveyors evaluating the same subjects for VAP. ${ }^{3-6}$ Furthermore, there is no consensus on how best to combine these and other criteria to identify VAP. One recent study identified 6 different possible definitions for VAP and demonstrated that they each identify different sets of subjects and generate different VAP rates. ${ }^{7}$ Finally, VAP's subjectivity is compounded by low specificity. Many conditions are associated with poor oxygenation, radiographic infiltrates, and changes in secretions, including pulmonary edema, ARDS, thromboembolic disease, pulmonary contusions, and others. ${ }^{8}$ Different background rates of these conditions in an ICU can have a substantial impact on the observed VAP rate. ${ }^{9}$

These weaknesses of traditional VAP definitions make it difficult or impossible to compare VAP rates between institutions or even to compare VAP rates within a single institution over time. One cannot know whether lower VAP rates in one institution or in one period versus another period are due to stricter interpretations of subjective clinical signs, differences in the prevalence of common mimickers of VAP, or true differences in the frequency of bona fide pneumonias.

A further limitation of VAP definitions is their relatively low attributable mortality rate. Early investigations noted that subjects with VAP were much more likely to die than subjects without VAP and that the net mortality rate among VAP subjects could be as high as 50\%. These analyses were confounded, however, by failure to take into account the baseline expected mortality rate for patients who develop VAP, since patients with VAP tend to be among the sickest and most vulnerable of critically ill patients. Subsequent analyses that attempted to match subjects with VAP to similar subjects without VAP, to adjust for important comorbidities, and/or to employ novel statistical strategies to overcome these sources of bias generated much lower estimates of the attributable mortality of VAP. ${ }^{10-12}$ Furthermore, if VAP is only responsible for a small fraction of ICU mortality, this begs the question of whether focusing quality improvement efforts on VAP detection and prevention alone is the best way to improve outcomes for ventilated populations. ${ }^{13}$ What about all of the other common and morbid conditions that can develop in ventilated patients, such as ARDS, pulmonary edema, severe sepsis, thromboembolic disease, and others? Focusing on VAP alone devalues the importance of these events and the necessity of designing prevention programs to address all of these potential complications of mechanical ventilation, not just pneumonia. 
Table 1. Limitations of Ventilator-Associated Pneumonia as a Quality Metric

VAP surveillance definitions are complicated and subjective

VAP surveillance is time-consuming and frustrating

Very difficult to automate surveillance

Frequent disagreement between clinicians and infection preventionists on who does and does not have VAP

Both clinicians and infection preventionists often wrong relative to histology

High interobserver variability among and between both clinicians and infection preventionists

Easy to lower VAP rates by applying surveillance criteria more strictly Susceptible to gaming

Attributable mortality very low

Very few VAP prevention initiatives have achieved decreased length of stay or lower mortality rates

Focusing on VAP prevention alone only addresses a small fraction of the potential harms that can befall ventilated patients.

Difficult or impossible to compare VAP rates between institutions

$\overline{\mathrm{VAP}}=$ ventilator-associated pneumonia

Finally, focusing on VAP alone can generate a misleading sense of improvement when none may be present. The weaknesses of VAP definitions allow for the possibility that lower VAP rates may be due to changes in surveillance rather than due to true improvements in care. ${ }^{14}$ This is a major risk when evaluating quality improvement programs and prevention strategies in before-after studies or open label trials. VAP surveyors and/or investigators may subconsciously apply VAP criteria more strictly in the intervention period or study arm, leading to fewer observed VAPs despite no true change in disease incidence. ${ }^{15}$

Difficulty distinguishing bona fide pneumonias from colonization of the oropharynx or endotracheal tube alone further complicates the interpretation of VAP prevention studies and initiatives. This is particularly the case for interventions designed to decrease microbial burden in the oropharynx and/or endotracheal tube, such as oral chlorhexidine and silver coating of endotracheal tubes. These interventions can lower the frequency of positive endotracheal aspirates, which in turn leads to fewer perceived pneumonias, but neither intervention has been shown to decrease mean duration of mechanical ventilation, ICU stay, or mortality rates. ${ }^{16-18}$ Failure to impact these outcomes begs the question of whether these interventions prevented true pneumonias or if they simply decreased the frequency of false positive pneumonias. Indeed, oral care with chlorhexidine may even be harmful to patients: 2 meta-analyses of oral care with chlorhexidine found higher mortality rates among patients randomized to chlorhexidine. ${ }^{18,19}$

A
\begin{tabular}{|c|c|c|}
\hline Date & $\begin{array}{c}\text { Minimum } \\
\text { PEEP }\end{array}$ & $\begin{array}{c}\text { Minimum } \\
\mathrm{F}_{\mathrm{IO}_{2}}\end{array}$ \\
\hline July 1 & 10 & 100 \\
\hline July 2 & 5 & 60 \\
\hline July 3 & 5 & 40 \\
\hline July 4 & 5 & 40 \\
\hline July 5 & 5 & 40 \\
\hline July 6 & 8 & 50 \\
\hline July 7 & 8 & 60 \\
\hline July 9 & 8 & 60 \\
\hline July 10 & 5 & 40 \\
\hline July 11 & 5 & 50 \\
\hline July 12 & 5 & 40 \\
\hline
\end{tabular}

B
\begin{tabular}{|c|c|c|}
\hline Date & $\begin{array}{c}\text { Minimum } \\
\text { PEEP }\end{array}$ & $\begin{array}{c}\text { Minimum } \\
\mathrm{F}_{\mathrm{IO}_{2}}\end{array}$ \\
\hline July 1 & 10 & 100 \\
\hline July 2 & 7.5 & 60 \\
\hline July 3 & 5 & 40 \\
\hline July 4 & 5 & 40 \\
\hline July 5 & 5 & 40 \\
\hline July 6 & 10 & 60 \\
\hline July 7 & 8 & 60 \\
\hline July 9 & 8 & 60 \\
\hline July 10 & 5 & 40 \\
\hline July 11 & 5 & 50 \\
\hline July 12 & 5 & 40 \\
\hline
\end{tabular}

Fig. 1. Two examples demonstrating patterns of daily ventilator settings in patients with ventilator-associated events. Patient $A$ has a significant increase in daily minimum PEEP lasting for $\geq 2 \mathrm{~d}$ following $\geq 2 \mathrm{~d}$ of stable or decreasing daily minimum PEEP. Patient $\mathrm{B}$ has a significant increase in daily minimum $\mathrm{F}_{1 \mathrm{O}_{2}}$ lasting for $\geq 2 \mathrm{~d}$ following $\geq 2 \mathrm{~d}$ of stable or decreasing daily minimum $\mathrm{F}_{\mathrm{IO}_{2}}$.

\section{The Genesis of VAE Definitions}

VAE definitions were developed by a working group of professional society representatives convened by the Centers for Disease Control and Prevention (CDC) in 2011 and 2012. Their intent was to overcome many of the limitations of traditional VAP definitions, specifically to make surveillance more objective, to broaden the focus of surveillance beyond pneumonia alone, and to allow for the possibility of automating surveillance using electronic health data. VAE surveillance is designed to identify patients with respiratory deterioration after a period of stability or improvement. This is defined as at least 2 days of increased levels of ventilator support after at least 2 days of stable or decreasing ventilator support. Ventilator support is measured using patients' daily minimum PEEP and daily minimum $\mathrm{F}_{\mathrm{IO}_{2}}$. A sustained increase in the daily minimum PEEP of $\geq 3 \mathrm{~cm} \mathrm{H}_{2} \mathrm{O}$ or a sustained increase in the daily minimum $\mathrm{F}_{\mathrm{IO}_{2}}$ of $\geq 20$ points qualifies (Fig. 1). The definitions use patients' daily minimum ventilator settings in order to get patients' best values each day and to avoid noise attributable to transient events, such as suctioning the endotracheal tube, bronchoscopies, repositioning patients, transportation, and/or procedures.

VAE definitions follow from the recognition that there is no perfect or even good definition for pneumonia available using current routine diagnostic tools. All existing definitions have many subjective components, and none has been shown to be more accurate than another. ${ }^{20,21}$ All yield many false positives and false negatives relative to histological evaluation at autopsy. Rather than try to come up with a new VAP definition then, the working group suggested shifting the focus of surveillance away from pneumonia to complications of mechanical ventilation instead. In doing so, the working group was simultaneously 


\begin{tabular}{|c|c|c|c|c|c|c|c|c|c|}
\hline Date & $\begin{array}{c}\text { Minimum } \\
\text { PEEP }\end{array}$ & $\begin{array}{c}\text { Minimum } \\
\mathrm{F}_{\mathrm{IO}_{2}}\end{array}$ & $\begin{array}{c}\text { Minimum } \\
\text { Temp }\end{array}$ & $\begin{array}{c}\text { Maximum } \\
\text { Temp }\end{array}$ & $\begin{array}{c}\text { Minimum } \\
\text { WBC }\end{array}$ & $\begin{array}{c}\text { Maximum } \\
\text { WBC }\end{array}$ & $\begin{array}{c}\text { Antibiotic } \\
1\end{array}$ & $\begin{array}{c}\text { Antibiotic } \\
2\end{array}$ & $\begin{array}{c}\text { Antibiotic } \\
3\end{array}$ \\
\hline July 1 & 10 & 100 & & & & & & & \\
\hline July 2 & 5 & 60 & & & & & & & \\
\hline July 3 & 5 & 40 & & & & & & & \\
\hline July 4 & 5 & 40 & 98.4 & 99.6 & 6.9 & 6.9 & & & \\
\hline July 5 & 5 & 40 & 98.7 & 100.2 & 9.1 & 16.3 & & & \\
\hline July 6 & 8 & 50 & 99.3 & 102.3 & 17.0 & 18.8 & Vanco & Pip-tazo & Gentamicin \\
\hline July 7 & 8 & 60 & 99.6 & 101.5 & 14.1 & 14.1 & Vanco & Pip-tazo & Gentamicin \\
\hline July 9 & 8 & 60 & 97.9 & 100.2 & 11.1 & 11.1 & Vanco & Pip-tazo & \\
\hline July 10 & 5 & 40 & & & & & & Pip-tazo & \\
\hline July 11 & 5 & 50 & & & & & & Pip-tazo & \\
\hline July 12 & 5 & 40 & & & & & & Pip-tazo & \\
\hline
\end{tabular}

Fig. 2. An example of a patient with an infection-related ventilator-associated complication (IVAC). This patient has a fever, leukocytosis, and $\geq 4 \mathrm{~d}$ of new antibiotics occurring within $2 \mathrm{~d}$ of a ventilator-associated event. Vanco = vancomycin; pip-tazo = piperacillin/tazobactam; $\mathrm{WBC}=$ white blood cells.

able to sidestep the inherent limitations of current VAP diagnostics, broaden the focus of surveillance (and hence prevention) to include additional morbid complications of mechanical ventilation, and simplify the surveillance definition, since the intent is only to identify episodes of nosocomial respiratory deterioration, not pneumonia.

The VAE definition set includes subcriteria for infection-related ventilator-associated complications (IVACs) and possible ventilator-associated pneumonia (PVAP). IVAC criteria aim to identify the subset of VAEs that may be infection-related, as indicated by concurrent abnormal temperature or white blood cell counts and the initiation of $\geq 4$ days of new antibiotics (Fig. 2). PVAP aims to identify the subset of IVACs that may be pneumonias by identifying patients with concurrent pulmonary specimens with moderate or heavy amounts of organism growth or with less growth but moderate or many neutrophils on Gram stain (an objective marker of purulence). An example of PVAP is shown in Figure 3. Although the VAE definition set does include PVAP, there is no reason to believe that PVAP is any more (or less) accurate than any prior pneumonia definition. The sole benefit of PVAP is to provide a familiar entity to clinicians within the framework of VAE surveillance. Indeed, CDC explicitly notes that whereas VAE and IVAC are potentially suitable for public reporting and benchmarking, PVAP is not suitable, given that (1) there is no reason to think it overcomes the limited accuracy of other VAP definitions, and (2) hospitals vary widely in their practices around the acquisition and processing of pulmonary specimens, and hence PVAP rates are inherently unsuitable for comparisons between institutions. ${ }^{1}$

\section{Data Supporting the Potential Value of VAEs as a Quality Indicator}

There is no consensus on what constitutes a suitable quality indicator, but arguably 3 key criteria are 1) clinical importance, 2) capacity to support objective and efficient surveillance, and 3) preventability. An ideal metric should identify a common clinical condition associated with high morbidity or mortality. The metric should be amenable to efficient surveillance in order to avoid burdening hospitals with excessive demands for data collection and analysis. Surveillance efficiency includes the capacity to define the metric using objective clinical data that are readily available in existing electronic data systems and amenable to automated analysis. Most importantly, a good metric should help identify potential lapses in care that can inform tangible strategies to improve care. Better care should then lead to lower event rates and better outcomes for patients. Studies to date suggest that VAE definitions may fulfill many of these criteria.

\section{Clinical Importance}

VAEs appear to be clinically important events. They affect $5-10 \%$ of patients and have consistently been associated with longer duration of mechanical ventilation, longer hospital lengths of stay, more antibiotic usage, and higher mortality rates. ${ }^{22-32}$ Multiple studies suggest that patients who develop VAEs are approximately twice as likely to die compared with similar patients who do not develop VAEs. ${ }^{22-32}$ 


\begin{tabular}{|c|c|c|c|c|c|c|c|c|c|}
\hline Date & $\begin{array}{c}\text { Minimum } \\
\text { PEEP }\end{array}$ & $\begin{array}{c}\text { Minimum } \\
\mathrm{F}_{1 O_{2}}\end{array}$ & Temp & WBC & $\begin{array}{c}\text { Antibiotic } \\
1\end{array}$ & $\begin{array}{c}\text { Antibiotic } \\
2\end{array}$ & $\begin{array}{c}\text { EA/BAL } \\
\text { Polys }\end{array}$ & $\begin{array}{c}\text { EA/BAL } \\
\text { Epis }\end{array}$ & Culture \\
\hline July 1 & 10 & 100 & & & & & & & \\
\hline July 2 & 5 & 60 & & & & & & & \\
\hline July 3 & 5 & 40 & & & & & & & \\
\hline July 4 & 5 & 40 & 99.9 & 11.2 & & & & & \\
\hline July 5 & 5 & 40 & 102.7 & 14.6 & Linezolid & Imipenem & $4+$ & 0 & S. aureus $>10^{5}$ CFU/mD \\
\hline July 6 & 7 & 60 & 101.6 & 18.4 & Linezolid & Imipenem & & & \\
\hline July 7 & 8 & 60 & 100.7 & 15.6 & Linezolid & & & & \\
\hline July 9 & 8 & 60 & 100.0 & 12.7 & Linezolid & & & & \\
\hline July 10 & 5 & 40 & & & Linezolid & & & & \\
\hline July 11 & 5 & 50 & & & Linezolid & & & & \\
\hline July 12 & 5 & 40 & & & Linezolid & & & & \\
\hline
\end{tabular}

Fig. 3. An example of a patient with a possible ventilator-associated pneumonia (PVAP). This patient meets all of the criteria for an infection-related ventilator-associated complication (IVAC) (increase in ventilator settings, abnormal temperature or white blood cell count, and $\geq 4 \mathrm{~d}$ of new antibiotics) but also has a concurrent pulmonary specimen with evidence of purulence (4+ neutrophils) on Gram stain and a positive quantitative culture for a pathogenic organism. The combination of IVAC with concurrent evidence of purulence and/or a positive quantitative or semiquantitative culture meets the criteria for PVAP. WBC = white blood cell; EA/BAL = endotracheal aspirate/bronchoalveolar lavage; Polys = polymorphonuclear leukocytes; Epis = epithelial cells.

\section{Surveillance Efficiency}

VAE definitions were purposefully designed to be objective and suitable for electronic surveillance. VAE avoids the subjectivity inherent in VAP definitions, since it is based solely on trends in 2 data elements: daily minimum PEEP and daily minimum $\mathrm{F}_{\mathrm{IO}_{2}}$. These are both concrete measures that all observers ought to be able to agree upon (in contrast to classic VAP criteria that invite debates as to whether an infiltrate is new or whether the volume and consistency of patients' secretions have changed from one day to another, etc). An increasing number of hospitals now document ventilator settings in their electronic health record systems or in independent databases maintained by respiratory therapy departments. Some hospitals are also able to automatically download ventilator settings directly from their ventilators into their electronic health records. The key data elements for VAE detection are therefore increasingly available electronically, making full automation of VAE surveillance increasingly possible. Multiple hospitals have now published their experience successfully automating VAE case detection. ${ }^{27-}$ $29,31,33$

Workers from the Netherlands have pointed out that one can define daily minimum PEEP and $\mathrm{F}_{\mathrm{IO}_{2}}$ in different ways and that this in turn can have a large effect on case detection. ${ }^{28}$ Options include the lowest values derived from minute-to-minute ventilator settings, the lowest values derived from paper charts with hourly documentation, or the 10th percentile. The CDC subsequently clarified that a setting can only qualify as the daily minimum value if the patient is maintained at that setting for at least $60 \mathrm{~min}$.

\section{Capacity to Guide Improvements in Care (Preventability)}

VAE criteria appear to identify potential opportunities to improve care and outcomes for patients. This is not readily apparent at first blush insofar as VAE is a novel surveillance concept rather than a longstanding clinical concept. Clinicians do not have an immediate intuitive feel for what conditions trigger VAEs and hence how best to prevent them. An expanding body of literature, however, fills this gap. At least 4 studies have evaluated consecutive subjects with VAEs to try to identify the clinical conditions that led to increases in ventilator support. ${ }^{22,25,28,30}$ Findings from these studies are similar. The majority of VAEs are caused by pneumonia, fluid overload, atelectasis, and/or ARDS. The relative frequency of these conditions varies between studies, but generally pneumonia accounts for about $20-40 \%$ of VAEs, fluid overload for $30-40 \%$, atelectasis for $10-20 \%$, and ARDS for $10-20 \% .22,25,28,30$

Knowing which conditions typically trigger VAEs helps to inform the development of strategies to prevent VAEs. Clinicians can intuit prevention strategies based on their prior experience with these 4 conditions as well as draw upon an increasing number of studies that identify specific risk factors for VAEs and demonstrate strategies that can decrease the frequency of VAEs.

Case-control studies have identified benzodiazepines, opioids, fluid balance, and high tidal volumes as risk factors for VAEs. ${ }^{34,35}$ Interventional studies have helped to affirm that improvements in practice can lower both $\mathrm{VAE}$ rates and mean duration of mechanical ventilation. 
Muscedere and colleagues ${ }^{26,36}$ assessed the impact of a 2 -y educational program to increase the adoption of clinical practice guidelines for ventilated subjects in 11 ICUs in Canada and the United States. They achieved relatively modest improvements in the frequency of guideline concordant practices, yet the investigators were still able to detect a significant decrease in VAEs from $13.6 \%$ of subjects sampled at baseline to $9.7 \%$ of subjects sampled at the end of the study $(P=.05) \cdot{ }^{26}$ On multivariable analysis, spontaneous awakening trials and spontaneous breathing trials were potentially protective against VAEs.

The CDC Prevention Epicenters Wake Up and Breathe Collaborative further affirmed the responsiveness of VAEs to less sedation and earlier extubation. ${ }^{37}$ The collaborative included 12 ICUs affiliated with 7 hospitals. These ICUs collectively worked to increase the frequency of coordinated daily spontaneous awakening trials and spontaneous breathing trials in eligible subjects using an opt-out protocol for nurses and respiratory therapists. Significant increases in the frequency of spontaneous awakening trials, spontaneous breathing trials, and the percentage of spontaneous breathing trials performed off sedation were associated with significant reductions in VAEs and IVACs. Collaborative members were able to decrease their collective VAE rate from 9.7 to 5.2 events/100 episodes (adjusted odds ratio $0.63,95 \% \mathrm{CI} 0.42-0.97$ ) and their IVAC rate from 3.5 to 0.52 events 100 episodes (adjusted odds ratio $0.35,95 \%$ CI $0.17-0.71$ ). Importantly, the decrease in VAE rates was also accompanied by significant decreases in mean duration of mechanical ventilation (2.4 fewer days, 95\% CI 1.7-3.1), ICU stay (3.0 fewer days, 95\% CI 1.6-4.3), and hospital stay ( 6.3 fewer days, $95 \%$ CI 4.0-8.6).

Finally, Mekontso Dessap et al ${ }^{38}$ have demonstrated that restrictive fluid management can decrease VAE rates by as much as $50 \%$. These investigators randomized subjects ready for weaning from mechanical ventilation to daily B-type natriuretic peptide measures versus usual care. B-type natriuretic peptide is a biomarker for hypervolemia. Subjects with high B-type natriuretic peptide levels were managed using a depletive fluid strategy (less fluid in, more fluid out). Daily B-type natriuretic peptide level measurements and depletive fluid management were associated with a $50 \%$ decrease in VAEs ( $8.6 \%$ of subjects vs $17.8 \%$ of subjects, $P=.02$ ) and significantly more ventilator-free days.

\section{VAE Surveillance: A Patient Safety Opportunity}

All told, the adoption of VAE as a quality metric carries substantial promise to improve outcomes for ventilated populations. ${ }^{39,40}$ VAE surveillance brings to light a broad group of patients suffering morbid complica- tions of mechanical ventilation beyond those identified through VAP surveillance alone. VAE analyses suggest promising strategies to improve care for ventilated patients. ${ }^{41}$ The potential interventions likely to prevent VAEs are highly aligned with emerging best practices in critical care. ${ }^{42-44}$ Finally, VAE surveillance is objective and amenable to automation, making it a more rigorous and efficient means to track the impact of care improvement programs compared with VAP surveillance.

\section{Concerns About the Potential Value of VAEs as a Quality Indicator}

Observers have raised important concerns about the potential suitability of VAE as a quality indicator. Critics cite the following issues: (1) the positive predictive value of VAEs for pneumonia is low, (2) the sensitivity of VAE for pneumonia is low, (3) VAE surveillance is susceptible to gaming, and (4) VAEs are probably just markers of severe illness rather than complications per se, and most are not preventable.

\section{The Positive Predictive Value of VAEs for Pneumonia Is Low}

A number of investigators have noted that the positive predictive value of VAEs for traditionally defined VAP is low. Most authors have reported that only about a quarter to a third of VAEs meet traditional surveillance definitions for VAP. ${ }^{22,26,28}$ Some investigators have even found that as few as $7 \%$ of VAEs meet VAP criteria. ${ }^{29}$ The fact that only a fraction of VAEs qualify as pneumonias, however, should not be a surprise, given that VAE definitions were specifically designed to broaden the purview of surveillance beyond pneumonia alone. The low positive predictive value then is expected and is arguably a strength of VAE insofar as it indicates that VAE surveillance is bringing to light morbid complications of mechanical ventilation beyond just pneumonia, such as fluid overload and ARDS.

\section{The Sensitivity of VAE Definitions for VAP Is Low}

Many studies have also pointed out that VAE surveillance misses many patients with traditionally defined VAPs. Indeed, in most series, VAE surveillance misses about $65-75 \%$ of traditionally defined VAPs. ${ }^{26,29,45,46}$ These false-negative cases merit contemplation.

First, it is worth recalling that VAE criteria were born out of the recognition that traditional VAP criteria are too subjective and inaccurate to produce meaningful benchmarks. Clinical audits suggest that up to $75 \%$ of patients treated for VAP most likely do not have pneumonia. ${ }^{47}$ 
Autopsy series also find that $40-60 \%$ of subjects who meet clinical criteria for VAP do not have histological evidence of pneumonia. ${ }^{21}$ VAP is therefore a questionable standard against which to measure the accuracy of VAE definitions.

Second, by definition, a VAP that does not meet criteria for VAE either did not have a period of stability or improvement before the pneumonia or did not require a sustained increase in ventilator settings equal to or greater than VAE thresholds ( $3 \mathrm{~cm}$ of PEEP or 20 points of $\mathrm{F}_{\mathrm{IO}_{2}}$ ). In one case series in a trauma ICU, 39\% of pneumonias missed by VAE were due to the lack of a period of stability or improvement. ${ }^{46}$ Many of these patients had pulmonary contusions and/or rib fractures, a population at high risk for pneumonia but also a population in which the diagnosis of pneumonia is particularly difficult, given the overlap in clinical signs between contusion, hemorrhage, ARDS, and pneumonia. Almost all of the remaining pneumonias missed by VAE criteria were patients who did not require sustained increases in ventilator settings. One wonders about the clinical importance of pneumonias that require little or no extra respiratory support. Some of these may have been mild pneumonias that improved very rapidly. Others may not have been pneumonias at all but perhaps alternative diagnoses or bacterial colonization alone.

Third, it is important to reiterate that VAE is a surveillance concept and not a clinical diagnosis. Surveillance is governed by a different set of values compared with clinical diagnosis. Clinical care, particularly in intensive care settings, places a very high value on sensitivity, given the potentially catastrophic consequences of missed or delayed diagnoses. Surveillance, by contrast, favors objectivity, reproducibility, and morbidity, since surveillance is only meaningful if it allows for comparisons over time and if it identifies severe events worth preventing. Focusing on more severe events decreases subjectivity, since severe events tend to be less ambiguous and more clearly meaningful. For example, workers trying to prevent surgical site infections tend to focus on deep and organ space infections rather than on superficial infections. ${ }^{48}$ Similarly, surveillance efforts to support better sepsis care tend to focus on patients with severe sepsis and septic shock rather than patients with systemic inflammatory response syndrome or sepsis alone. In the same vein, VAE criteria focus surveillance on the subset of particularly severe and unambiguous pneumonias that require sustained increases in ventilator support. At the end of the day, however, VAE analyses by their nature are designed to identify care improvement opportunities that can be applied to the care of all patients in the ICU. Hence changes in care learned from analyses of triggers for severe pneumonias should also help to prevent milder pneumonias, even if they would never have triggered VAE criteria.

\section{VAE Surveillance Is Susceptible to Gaming}

Two papers have suggested strategies for gaming VAE surveillance. One group suggested managing patients with high ventilator settings throughout their time on mechanical ventilation, since this will preclude the need to increase ventilator settings in response to new clinical events. ${ }^{49}$ The problem with this strategy is that it is clinically dangerous, since sustained high PEEP and/or hyperoxemia are both independent risk factors for harm..$^{50} \mathrm{An}$ other group suggested alternately raising and lowering the PEEP by $1 \mathrm{~cm} \mathrm{H}_{2} \mathrm{O}$ each day. This will eliminate the possibility of a stable baseline and therefore preclude any VAEs. ${ }^{29}$ Both strategies, however, could arguably be construed as fraud. If VAE ever does become a quality indicator, then presumably health departments, accrediting agencies, and/or payers will review hospitals' primary ventilator data periodically to assure compliance. Hospitals that appear to be deliberately adjusting ventilator settings solely to avoid VAE detection could face penalties.

Nonetheless, these two suggestions on avoiding VAEs underscore the fact that VAE surveillance is not immune to gaming and bias. Clinicians do have the capacity to prevent VAE detection by manipulating ventilator settings. Traditional VAP surveillance was also susceptible to missing cases depending on clinician behavior (eg, opting not to order chest radiographs or culture specimens). The big difference in bias risk between VAE and VAP surveillance is that VAP surveillance was subject to bias from both clinicians and surveyors. VAE surveillance, by contrast, eliminates infection preventionists' scope for subjective decision making, since the VAE definitions are strictly mathematical.

\section{Most VAEs Are Not Preventable}

Commentators have suggested that VAEs are merely surveillance markers for severe disease rather than complications per se..$^{2,49,51}$ Boyer et al ${ }^{30}$ evaluated all VAEs detected in their institution over a 1-y period and tried to determine whether each event was preventable. They estimated that only $37 \%$ of VAEs were potentially preventable. Adjudicating preventability, however, is notoriously difficult. Although the immediate event that precipitated the VAE may not have appeared to be preventable, it is impossible to know what could have happened if the patient had been managed differently from their first day of mechanical ventilation. Perhaps with less sedation and earlier mobilization, the patient may have been extubated long before the supposedly inevitable event. Preventability is better assessed through prospective intervention studies, and, as outlined above, at least 3 studies thus far have demonstrated successful decreases in VAE rates. $26,37,38$ 


\section{VAEs AS A QUALITY INDICATOR IN THE ICU}

\section{The Con Position}

\section{The VAE Algorithm Is Confusing}

Since the initial development of a VAE algorithm in 2013, the National Health Safety Network has made several modifications to the algorithm aimed at improving VAE surveillance. The new VAE algorithm has three definitional tiers: ventilator-associated conditions (VAC), IVAC, and PVAP (Figs. 1-3). The first tier of the algorithm, VAC, only takes into account changes in patients' ventilator settings. The 2 subsequent tiers of the VAE algorithm, IVAC and PVAP, require that patients must first meet the initial VAC definition. However, there is a clear conceptual flaw with all 3 tiers insofar as they are all only apparent $\geq 48 \mathrm{~h}$ after respiratory deterioration first occurs. Thus, although the VAE algorithm is not intended for diagnostic use by clinicians, its after-the-fact nature is so divorced from clinical logic that it is clearly not useful to practicing ICU physicians within the normative time course of care.

\section{VAE Surveillance Is Insensitive for Identifying VAP}

An ideal ICU quality indicator should have both high sensitivity and high specificity and thus must be compared with some accepted standard of measurement. For VAE, the accepted standard of comparison is arguably VAP. Many studies have noted, however, that VAE surveillance misses many pneumonias. Klein Klouwenberg et al, ${ }^{28}$ for example, found that the VAE algorithm detected $33 \%$ of VAPs at most. Likewise, most VAEs were caused by volume overload and infections but not necessarily VAP. Indeed, the positive predictive value of a VAE for VAP was only $25 \%$ (95\% CI $18-33 \%$ ). Klein Klouwenberg et al ${ }^{28}$ further reported that, even in cases that were positive for VAC or IVAC as well as clinically defined VAP, these events did not necessarily occur at the same time. Lilly et al, ${ }^{29}$ investigating 8,408 mechanically ventilated subjects, also demonstrated that VAE/VAC detected less than a third of VAP cases, with a sensitivity of $32.5 \%$ and a positive predictive value of $7 \%$. A third study in trauma subjects requiring $>48 \mathrm{~h}$ of ventilation similarly reported that IVAC criteria had low accuracy in identifying VAP in high-risk trauma subjects..$^{52}$ The sensitivity and positive predictive values of IVAC for VAP identification were 28.1 and $58.6 \%$, respectively, meaning that the algorithm failed to detect about three quarters of subjects with VAP.

The VAE algorithm detects a broad spectrum of conditions and complications associated with changes in ventilator settings that correspond to the VAC criteria, the most common of which are VAP, ARDS, pulmonary edema, and atelectasis. ${ }^{22}$ In a retrospective study by
Hayashi et al,25 the authors assessed the etiology of VAE, finding that among 143 subjects with VAE, 30.7\% had microbiological evidence of VAP. Another 31\%, however, had no microbiological evidence of VAP. Other etiologies that could have explained VAE included: atelectasis in $16.3 \%$ of subjects, acute pulmonary edema in $11.8 \%$, ARDS in $6.5 \%$, pleural effusion in $3 \%$, pulmonary embolism in $2 \%$, and abdominal distention in $1.3 \%$. Lilly et $\mathrm{al}^{29}$ found that ARDS was associated with $73 \%$ of VAE cases and that acute kidney injury was associated with $27 \%$. Given that most VAEs are not VAPs, the definition will cause confusion among physicians and will be difficult to implement for the purpose of significant clinical surveillance.

\section{VAE Surveillance Is Not a Comprehensive Assessment of a Physiological System}

An ICU quality indicator should be universally applicable and easy to use for clinicians, researchers, and administrators for characterizing patients in terms of severity of illness. The Acute Physiology and Chronic Health Evaluation (APACHE) along with the Risk, Injury, Failure, Loss, and End-stage kidney classification (RIFLE) are the most frequently used general severity-of-illness and renal failure severity scores in adult ICU patients. These score systems seek to take into account the entire range of relevant and available clinical data to predict patient outcomes. ${ }^{53,54}$ The applicability and universality of both the APACHE and RIFLE systems have been well tested and demonstrated by comprehensive multi-center large sample studies. ${ }^{55,56}$ However, there is still debate regarding the rationale for using VAE in the ICU, and further multicenter prospective validation studies are needed. Furthermore, VAE is not a scoring system for an organ or functional system in the body. As previously mentioned, the first qualifying tier of VAE is entirely based on changes in ventilator settings (specifically PEEP and $\mathrm{F}_{\mathrm{IO}_{2}}$ ), and these changes do not necessarily represent changes in patients' pulmonary status. An increased APACHE or RIFLE score is associated with increased mortality, whereas a diagnosis of VAE might not always be associated with increased mortality because it can sometimes be indicative of what could actually be improved care, such as raising PEEP in the setting of atelectasis.

\section{VAE Surveillance Is Susceptible to Abuse and Misuse}

Since the first qualifying tier of the VAE algorithm, VAC, concerns changes in the ventilator settings of $\mathrm{F}_{\mathrm{IO}_{2}}$ and PEEP, the VAE algorithm is susceptible to manipulation. Lilly et al, ${ }^{29}$ for example, reported that VAE/VAC rates can be reduced $93 \%$ by easy manipulation of ventilator management protocols. Thus, the new VAE algo- 
Table 2. Pros and Cons of Ventilator-Associated Events as a Quality Metric

\begin{tabular}{|c|c|}
\hline Pros & Cons \\
\hline $\begin{array}{l}\text { VAE definitions are objective, reproducible, electronically } \\
\text { computable, and amenable to automation. }\end{array}$ & $\begin{array}{l}\text { VAE is an unfamiliar entity to most clinicians (no gestalt sense as to } \\
\text { what it means). }\end{array}$ \\
\hline $\begin{array}{l}\text { First tier of the VAE framework is conceptually very simple to define } \\
\text { and explain (change in PEEP or } \mathrm{F}_{\mathrm{IO}_{2}} \text { ). }\end{array}$ & $\begin{array}{l}\text { The second and third tiers of the VAE framework (IVAC and } \\
\text { PVAP) are complicated to explain and automate. }\end{array}$ \\
\hline VAEs strongly and consistently associated with increased mortality. & VAE surveillance misses many traditionally defined VAPs. \\
\hline \multirow{2}{*}{$\begin{array}{l}\text { VAE definitions expand the focus of surveillance and prevention to } \\
\text { include multiple causes of deterioration in ventilated patients, not } \\
\text { just pneumonia. }\end{array}$} & Positive predictive value of VAE for VAP is low. \\
\hline & $\begin{array}{l}\text { Ventilator settings are poor and indirect measures of respiratory } \\
\text { physiology. }\end{array}$ \\
\hline \multirow{2}{*}{$\begin{array}{l}\text { There is mounting evidence that VAEs can be prevented and that } \\
\text { preventing VAEs is associated with less time to extubation and } \\
\text { other objective benefits. }\end{array}$} & Sometimes raising ventilator settings can be good for patients. \\
\hline & $\begin{array}{l}\text { Some VAEs may better reflect the natural history of severe illness } \\
\text { rather than potentially preventable complications. }\end{array}$ \\
\hline $\begin{array}{l}\text { Best practices to prevent VAEs highly aligned with best practices in } \\
\text { critical care. }\end{array}$ & $\begin{array}{l}\text { VAE surveillance requires infection preventionists to grapple with } \\
\text { new sources of data (ventilator settings) and practices of care }\end{array}$ \\
\hline \multirow{4}{*}{$\begin{array}{l}\text { VAEs provide a tangible focus for root cause analyses to explore } \\
\text { local factors that may be modifiable to improve outcomes for } \\
\text { ventilated patients. } \\
\text { VAE surveillance and prevention encourages cross-collaboration } \\
\text { between multiple disciplines (physicians, nurses, infection control, } \\
\text { respiratory therapy, pharmacy, physical therapy, etc). }\end{array}$} & (ventilator management, sedation management, fluid management) \\
\hline & VAE detection can be averted by manipulating ventilator settings in \\
\hline & trivial ways. \\
\hline & $\begin{array}{l}\text { Extent of VAE preventability unknown. It is unlikely that all VAEs } \\
\text { can be avoided. }\end{array}$ \\
\hline \multicolumn{2}{|l|}{ VAE $=$ ventilator-associated event } \\
\hline \multicolumn{2}{|l|}{$\mathrm{VAP}=$ ventilator-associated pneumonia } \\
\hline \multicolumn{2}{|l|}{$\begin{array}{l}\text { IVAC }=\text { infection-related ventilator-associated complication } \\
\text { PVAP }=\text { possible ventilator-associated pneumonia }\end{array}$} \\
\hline
\end{tabular}

rithm can easily be gamed to suggest improved ICU quality of care. If VAE surveillance is subsequently linked to public reporting, pay-for-performance, or data-driven incentive policies, physicians will be inclined to manipulate patients' ventilator strategies to minimize the incidence of VAE.

Furthermore, a lack of changes in ventilator settings is not equivalent to stable patient health, and vice versa. Changes in ventilator settings also have a close relationship to the therapeutic approach of the caregivers. Due to the diversity of detailed ventilator management strategies, different ICU teams may have different $\mathrm{F}_{\mathrm{IO}_{2}}$ and PEEP protocols for a patient on mechanical ventilation. The VAE algorithm is in truth a reflection of caregiver tendencies rather patient condition. This is one of the most important reasons why such a ventilator setting-oriented algorithm cannot be used as a health-care quality indicator in the ICU.

\section{VAEs Can Sometimes Be Triggered by Improvements in Care}

The VAE algorithm focuses on increases in ventilator settings during mechanical ventilation. However, when a patient is obese or suffering from fluid overload, clinicians generally make significant PEEP and $\mathrm{F}_{\mathrm{IO}_{2}}$ changes in order to improve care. Also, the more aggressive the attempts are to liberate the patient from the ventilator, the more likely failed extubation becomes, consequently leading to a higher likelihood of increased PEEP and $\mathrm{F}_{\mathrm{IO}_{2}}$ and thus a higher incidence of VAE. Therefore, if VAE becomes a universally adopted form of ICU quality surveillance, clinicians might be less inclined to extubate mechanically ventilated patients. Paradoxically, this could lead to a situation in which VAP incidence increases due to prolonged ventilatory times and VAE incidence decreases because of fewer failed extubations. ${ }^{57}$

\section{Con Position Concluding Remarks}

The primary aim of the new VAE algorithm was to create a more objective, efficient, and reliable measure of complications associated with mechanical ventilation compared with the current clinician-dependent assessment of VAP occurrence. ${ }^{1,4}$ However, VAE surveillance does not perform as well as VAP surveillance at detecting VAP and has several undesirable characteristics that could make its implementation as an ICU quality indicator difficult. The VAE algorithm has a low sensitivity and positive predictive value for identifying VAP, and there are difficulties in standardizing the implementation of VAE surveillance due to easily exploitable loopholes in its definition. The VAE algorithm needs significant modifications and improvement in order to replace VAP as a surveillance method and quality indicator in the ICU. The preliminary data on the 


\section{VAEs AS A QUALITY INDICATOR IN THE ICU}

topic must be confirmed in additional large prospective studies.

\section{Conclusions}

Ventilator-associated events are still very new concepts for clinicians, administrators, and policy makers. Pros and cons of VAE as a potential quality improvement metric are summarized in Table 2. There is a substantial body of work demonstrating the feasibility of electronic surveillance and the high attributable morbidity and mortality of VAEs. A smaller number of emerging studies are helping to identify risk factors for VAEs and to demonstrate tangible strategies for prevention. Nonetheless, there is still much to be learned about the full extent to which VAEs are preventable and whether they can meaningfully influence hospital quality improvement programs for the better. Concerns about VAE's complexity, limited overlap with VAP, and potential to be gamed persist. More data on preventability and more consensus on utility are needed if VAE is ever to serve as a quality indicator for ICUs.

\section{REFERENCES}

1. Magill SS, Klompas M, Balk R, Burns SM, Deutschman CS, Diekema D, et al. Developing a new, national approach to surveillance for ventilator-associated events. Crit Care Med 2013; 41(11):2467-2475.

2. Lilly CM, Ellison RT 3rd. Quality measures for critically ill patients: where does ventilator-associated condition fit in? Chest 2013;144(5): 1429-1430.

3. Schurink CA, Van Nieuwenhoven CA, Jacobs JA, Rozenberg-Arska $\mathrm{M}$, Joore HC, Buskens E, et al. Clinical pulmonary infection score for ventilator-associated pneumonia: accuracy and inter-observer variability. Intensive Care Med 2004;30(2):217-224.

4. Klompas M. Interobserver variability in ventilator-associated pneumonia surveillance. Am J Infect Control 2010;38(3):237-239.

5. Stevens JP, Kachniarz B, Wright SB, Gillis J, Talmor D, Clardy P, Howell MD. When policy gets it right: variability in U.S. hospitals' diagnosis of ventilator-associated pneumonia. Crit Care Med 2014; 42(3):497-503.

6. Klein Klouwenberg PM, Ong DS, Bos LD, de Beer FM, van Hooijdonk RT, Huson MA, et al. Interobserver agreement of Centers for Disease Control and Prevention criteria for classifying infections in critically ill patients. Crit Care Med 2013;41(10): 2373-2378.

7. Ego A, Preiser JC, Vincent JL. Impact of diagnostic criteria on the incidence of ventilator-associated pneumonia. Chest 2015;147(2): 347-355.

8. Petersen IS, Aru A, Skødt V, Behrendt N, Bols B, Kiss K, Simonsen $\mathrm{K}$. Evaluation of pneumonia diagnosis in intensive care patients. Scand J Infect Dis 1999;31(3):299-303.

9. Klompas M, Kulldorff M, Platt R. Risk of misleading ventilatorassociated pneumonia rates with use of standard clinical and microbiological criteria. Clin Infect Dis 2008;46(9):1443-1446.

10. Muscedere JG, Day A, Heyland DK. Mortality, attributable mortality, and clinical events as end points for clinical trials of ventilatorassociated pneumonia and hospital-acquired pneumonia. Clin Infect Dis 2010;51(Suppl 1):S120-S125.
11. Nguile-Makao M, Zahar JR, Français A, Tabah A, GarrousteOrgeas M, Allaouchiche B, et al. Attributable mortality of ventilator-associated pneumonia: respective impact of main characteristics at ICU admission and VAP onset using conditional logistic regression and multi-state models. Intensive Care Med 2010;36(5): 781-789.

12. Melsen WG, Rovers MM, Groenwold RH, Bergmans DC, Camus C, Bauer TT, et al. Attributable mortality of ventilator-associated pneumonia: a meta-analysis of individual patient data from randomised prevention studies. Lancet Infect Dis 2013;13(8):665-671.

13. Klompas M, Li L. Beyond pneumonia: improving care for ventilated patients. Lancet Infect Dis 2013;13(8):640-641.

14. Klompas M. Eight initiatives that misleadingly lower ventilator-associated pneumonia rates. Am J Infect Control 2012;40(5):408-410.

15. Klompas $\mathbf{M}$. The paradox of ventilator-associated pneumonia prevention measures. Crit Care 2009;13(5):315.

16. Kollef MH, Afessa B, Anzueto A, Veremakis C, Kerr KM, Margolis $\mathrm{BD}$, et al. Silver-coated endotracheal tubes and incidence of ventilator-associated pneumonia: the NASCENT randomized trial. JAMA 2008;300(7):805-813.

17. Klompas M. Silver-coated endotracheal tubes and patient outcomes in ventilator-associated pneumonia. JAMA 2008;300(22):2605; author reply 2605-2606.

18. Klompas M, Speck K, Howell MD, Greene LR, Berenholtz SM. Reappraisal of routine oral care with chlorhexidine gluconate for patients receiving mechanical ventilation: systematic review and meta-analysis. JAMA Intern Med 2014;174(5):751-761.

19. Price R, MacLennan G, Glen J. Selective digestive or oropharyngeal decontamination and topical oropharyngeal chlorhexidine for prevention of death in general intensive care: systematic review and network meta-analysis. BMJ 2014;348:g2197.

20. Klompas M. Does this patient have ventilator-associated pneumonia? JAMA 2007;297(14):1583-1593.

21. Tejerina E, Esteban A, Fernández-Segoviano P, Frutos-Vivar F, Aramburu J, Ballesteros D, Rodríguez-Barbero JM. Accuracy of clinical definitions of ventilator-associated pneumonia: comparison with autopsy findings. J Crit Care 2010;25(1):62-68.

22. Klompas M, Khan Y, Kleinman K, Evans RS, Lloyd JF, Stevenson $\mathrm{K}$, et al. Multicenter evaluation of a novel surveillance paradigm for complications of mechanical ventilation. PLoS One 2011;6(3): e18062.

23. Klompas M, Magill S, Robicsek A, Strymish JM, Kleinman K, Evans RS, et al. Objective surveillance definitions for ventilatorassociated pneumonia. Crit Care Med 2012;40(12):3154-3161.

24. Prospero E, Illuminati D, Marigliano A, Pelaia P, Munch C, Barbadoro P, D'Errico MM. Learning from Galileo: ventilator-associated pneumonia surveillance. Am J Respir Crit Care Med 2012;186(12): 1308-1309.

25. Hayashi Y, Morisawa K, Klompas M, Jones M, Bandeshe H, Boots R, et al. Toward improved surveillance: the impact of ventilator-associated complications on length of stay and antibiotic use in patients in intensive care units. Clin Infect Dis 2013; 56(4):471-477.

26. Muscedere J, Sinuff T, Heyland DK, Dodek PM, Keenan SP, Wood $\mathrm{G}$, et al. The clinical impact and preventability of ventilator-associated conditions in critically ill mechanically ventilated patients. Chest 2013;144(5): 1453-1460.

27. Klompas M, Kleinman K, Murphy MV. Descriptive epidemiology and attributable morbidity of ventilator-associated events. Infect Control Hosp Epidemiol 2014;35(5):502-510.

28. Klein Klouwenberg PM, van Mourik MS, Ong DS, Horn J, Schultz MJ, Cremer OL, et al. Electronic implementation of a novel surveillance paradigm for ventilator-associated events: feasibility and validation. Am J Respir Crit Care Med 2014;189(8):947-955. 


\section{VAEs AS A QUALITY INDICATOR IN THE ICU}

29. Lilly CM, Landry KE, Sood RN, Dunnington CH, Ellison RT 3rd, Bagley $\mathrm{PH}$, et al. Prevalence and test characteristics of National Health Safety Network ventilator-associated events. Crit Care Med 2014;42:2019-2028.

30. Boyer AF, Schoenberg N, Babcock H, McMullen KM, Micek ST, Kollef MH. A prospective evaluation of ventilator-associated conditions and infection-related ventilator-associated conditions. Chest 2015;147(1):68-81.

31. Stevens JP, Silva G, Gillis J, Novack V, Talmor D, Klompas M, Howell MD. Automated surveillance for ventilator-associated events. Chest 2014;146(6):1612-1618.

32. Bouadma L, Sonneville R, Garrouste-Orgeas M, Darmon M, Souweine B, Voiriot G, et al. Ventilator-associated events: prevalence, outcome, and relationship with ventilator-associated pneumonia. Crit Care Med 2015;43(9):1798-1806.

33. Resetar E, McMullen KM, Russo AJ, Doherty JA, Gase KA, Woeltje KF. Development, implementation and use of electronic surveillance for ventilator-associated events (VAE) in adults. AMIA Annu Symp Proc 2014;2014:1010-1017.

34. Lewis SC, Li L, Murphy MV, Klompas M, CDC Prevention Epicenters. Risk factors for ventilator-associated events: a case-control multivariable analysis. Crit Care Med 2014;42(8):1839-1848.

35. Ogbu OC, Martin GS, Sevransky JE, Murphy DJ. High tidal volumes are independently associated with development of a ventilator-associated condition in the ICU. Am J Respir Crit Care Med 2015;191: A3117.

36. Sinuff T, Muscedere J, Cook DJ, Dodek PM, Anderson W, Keenan $\mathrm{SP}$, et al. Implementation of clinical practice guidelines for ventilator-associated pneumonia: a multicenter prospective study. Crit Care Med 2013;41(1):15-23

37. Klompas M, Anderson D, Trick W, Babcock H, Kerlin MP, Li L, et al. The preventability of ventilator-associated events: the CDC Prevention Epicenters Wake Up and Breathe Collaborative. Am J Respir Crit Care Med 2015;191(3):292-301.

38. Mekontso Dessap A, Katsahian S, Roche-Campo F, Varet H, Kouatchet A, Tomicic V, et al. Ventilator-associated pneumonia during weaning from mechanical ventilation: role of fluid management. Chest 2014;146(1):58-65.

39. Klompas M. Complications of mechanical ventilation: the CDC's new surveillance paradigm. N Engl J Med 2013;368(16):1472-1475.

40. Klompas M. Ventilator-associated events surveillance: a patient safety opportunity. Curr Opin Crit Care 2013;19(5):424-431.

41. Klompas M. Potential strategies to prevent ventilator-associated events. Am J Respir Crit Care Med 2015;192(12):1420-1430.

42. Barr J, Fraser GL, Puntillo K, Ely EW, Gélinas C, Dasta JF, et al. Clinical practice guidelines for the management of pain, agitation, and delirium in adult patients in the intensive care unit. Crit Care Med 2013;41(1):263-306.

43. Halpern SD, Becker D, Curtis JR, Fowler R, Hyzy R, Kaplan LJ, et al. An official American Thoracic Society/American Association of Critical-Care Nurses/American College of Chest Physicians/Society of Crit Care Med policy statement: the Choosing Wisely Top 5 list in Crit Care Med Am J Respir Crit Care Med 2014;190(7):818-826.

44. Morandi A, Brummel NE, Ely EW. Sedation, delirium and mechanical ventilation: the "ABCDE" approach. Curr Opin Crit Care 2011; 17(1):43-49.

45. Chang HC, Kung SC, Wang CM, Liu WL. Discordance between novel and traditional surveillance paradigm of ventilator-associated pneumonia. Infect Control Hosp Epidemiol 2014;35(9):1195-1196.

46. Stoeppel CM, Eriksson EA, Hawkins K, Eastman A, Wolf S, Minei J, Minshall CT. Applicability of the National Healthcare Safety Network's surveillance definition of ventilator-associated events in the surgical intensive care unit: a 1-year review. J Trauma Acute Care Surg 2014;77(6):934-937.

47. Nussenblatt V, Avdic E, Berenholtz S, Daugherty E, Hadhazy E, Lipsett PA, et al. Ventilator-associated pneumonia: overdiagnosis and treatment are common in medical and surgical intensive care units. Infect Control Hosp Epidemiol 2014;35(3):278-284.

48. Anderson DJ, Chen LF, Sexton DJ, Kaye KS. Complex surgical site infections and the devilish details of risk adjustment: important implications for public reporting. Infect Control Hosp Epidemiol 2008; 29(10):941-946.

49. Niederman MS, Nair GB. Managing ventilator complications in a "VACuum" of data. Chest 2015;147(1):5-6.

50. Helmerhorst HJ, Roos-Blom MJ, van Westerloo DJ, de Jonge E. Association between arterial hyperoxia and outcome in subsets of critical illness: a systematic review, metaanalysis, and meta-regression of cohort studies. Crit Care Med 2015;43(7):1508-1519.

51. Shorr AF, Zilberberg MD. Nature (and the ICU) abhors a VACuum. Chest 2012;142(6):1365-1366.

52. Piriyapatsom A, Lin H, Pirrone M, De Pascale G, Corona De Lapuerta J, Bittner EA, et al. Evaluation of the infection-related ventilatorassociated events algorithm for ventilator-associated pneumonia surveillance in a trauma population. Respir Care 2016:61(3):269-276.

53. Salluh JI, Soares M. ICU severity of illness scores: APACHE, SAPS and MPM. Curr Opin Crit Care 2014;20(5):557-565.

54. Hoste EA, Clermont G, Kersten A, Venkataraman R, Angus DC, De Bacquer D, Kellum JA. RIFLE criteria for acute kidney injury are associated with hospital mortality in critically ill patients: a cohort analysis. Crit Care 2006;10(3):R73.

55. Zimmerman JE, Kramer AA, McNair DS, Malila FM. Acute Physiology and Chronic Health Evaluation (APACHE) IV: hospital mortality assessment for today's critically ill patients. Crit Care Med 2006;34(5):1297-1310.

56. Bagshaw SM, George C, Bellomo R, ANZICS Database Management Committee. A comparison of the RIFLE and AKIN criteria for acute kidney injury in critically ill patients. Nephrol Dial Transplant 2008;23(5):1569-1574.

57. Giard M, Lepape A, Allaouchiche B, Guerin C, Lehot JJ, Robert $\mathrm{MO}$, et al. Early- and late-onset ventilator-associated pneumonia acquired in the intensive care unit: comparison of risk factors. J Crit Care 2008;23(1):27-33.

\section{Discussion}

Klompas: Many have asked where the specific VAE threshold values for significant changes in PEEP ( $\geq 3 \mathrm{~cm} \mathrm{H} \mathrm{H}_{2} \mathrm{O}$ ) and $\mathrm{F}_{\mathrm{IO}_{2}}$ ( $\geq 20$ points) came from. They were suggested by a working group of critical care, infectious disease, health-care epidemiol- ogy, and public health stakeholders convened by the CDC. ${ }^{1}$ Interestingly, an earlier paper $^{2}$ (the first paper suggesting the concept of ventilator-associated condition) used a PEEP threshold of $\geq 2.5 \mathrm{~cm} \mathrm{H}_{2} \mathrm{O}$ and an $\mathrm{F}_{\mathrm{IO}_{2}}$ threshold of $\geq 0.15$. The CDC VAE working group, however, felt that these threshold changes were too small and therefore proposed slightly higher thresholds. There is a paper ${ }^{3}$ comparing the incidence and attributable morbidity and mortality of different thresholds for significant changes in PEEP and $\mathrm{F}_{\mathrm{IO}_{2}}$. They looked at PEEP thresholds of 2,3 , and $5 \mathrm{~cm}$ of $\mathrm{H}_{2} \mathrm{O}$ and $\mathrm{F}_{\mathrm{IO}_{2}}$ thresholds of 15 and 20 points. The paper noted a clear pattern: The higher the thresh- 
old, the fewer events. However, there were only marginal differences in attributable mortality between thresholds. So that's the genesis behind the threshold values. They're semi-evidencebased with an overlay of gestalt.

Hurford: Michael, What disease or event are you intending to prevent by tracking VAEs? So, if I reduce VAEs, do I really make anything better?

Klompas: VAE is a global metric designed to capture many different kinds of complications, not just one condition in particular. Experience to date, however, suggests that most VAEs are caused by one of 4 conditions: pneumonia, ARDS, atelectasis, and pulmonary edema. So VAE surveillance and whatever prevention programs it triggers have the greatest potential to prevent these events. Which of these events you're most likely to prevent will of course depend on which intervention strategy you choose. If you target better fluid management, you'll decrease the pulmonary edema component. If you choose low $V_{T}$ ventilation, you're going to decrease the ARDS component.

Hurford: Right. But one you're treating with diuretics and the other you're treating with antibiotics. How, physiologically, are you justified in lumping those 2 together other than coming up with some simple criteria to collect? This is sort of like the PSI 90 , this big glut of patient safety indicators in which you're supposedly better if you reduce them. I don't understand the goal here. What disease are we treating by tracking this? Edema? Atelectasis? Pneumonia? These are very different diseases.

Klompas: You're capturing all the above, and hence you have the potential to design VAE prevention programs that could prevent all the above. You can do so by implementing programs designed to target the 4 most common conditions that trigger VAEs (pneumonia, atelectasis, pulmonary edema, ARDS). Or, what I think you really should be doing, in addition, is root cause analyses for every VAE to try to understand what the causative factors were for each particular case and what could potentially be done differently for future patients. It might be that you'll find that the VAE was triggered by one of the conditions I've mentioned. Or maybe you'll find something else entirely, such as patient transport or maybe the cuff pressures you're using. I don't know; check it out. But it's an opportunity for you to take a deeper dive into the subset of individuals on your ICU who are suffering highly morbid complications.

Hurford: But duration of mechanical ventilation per patient would give you basically the same thing. If your patients are doing worse, you'll be able to tell that the patients are doing worse without tracking VAEs. And if you want to learn fluid balance, why not just measure fluid balance?

Klompas: Mean duration of mechanical ventilation is attractive and is clearly very clinically meaningful. The problem is that for any given patient, you won't know whether their mean duration of mechanical ventilation is good or bad, right? Because mean duration of mechanical ventilation is primarily driven by whatever condition led to the patient being placed on mechanical ventilation and by their severity of illness. A long duration of mechanical ventilation might be very reasonable for a given individual. VAE, by contrast, says something went wrong with this individual, they had a trajectory change in their ventilator support, look more closely at this event and try to understand where the opportunities lie. It helps to focus on a particular event in a particular patient where you know there was a problem for that patient as opposed to mean duration of mechanical ventilation, where there may or may not have been something wrong. Your second question was: Should we just be putting into place a way to track patients' fluid balances and other processes of care? Yes, you prob- ably should be doing that. If you do VAE surveillance and you identify that fluids remain a problem in your unit, you probably will do that and maybe bring others to the same conclusion you intuited. But what about all the other potential opportunities that might lie there? Are you doing full mobilization on all your patients to the maximum extent possible? Have you really got your patients down to a minimum amount of sedation? VAE is your opportunity to try and understand that more closely.

Kallet: One of the slides that I found disturbing, and it might have been somewhat flippant, was about trying to prevent gaming the system. The chart showed increasing the PEEP by 1 and then the big label FRAUD across there. Given my recent experience, it's hard for me to talk about VAP and benchmarking without getting apoplectic in a couple of minutes. Case in point, I'm chair of my hospital's task force on VAP and the administration went absolutely nuts because out of 1,110 ventilator cases in 1 year we had 10 VAP cases by our surveillance data. They demanded to know: How are you going to get this to 0 ? And everyone was terrorized about this supposed problem. The reason we're having this discussion is that the government has threatened to punish hospitals for this. And in terms of making this part of hospital accreditation or not reimbursing through Medicare, I think drives a lot of this. We're all in agreement that we want to minimize VAP, but I think how this has played out has been hugely negative. So when I see something like, "Oh, if you just go up 1 on the PEEP or if you did a recruitment maneuver to try to bring that VAE rate down, that would be considered fraud," I find it disturbing. Although I think you were being a little flippant with that example, someone in government with the power to impose draconian measures might take that very seriously.

Klompas: Some wise person said to me, you can either do quality improve- 
ment or you can do public reporting/ benchmarking/pay-for-performance. These are actually contradictory things because the moment you put out the specter of benchmarking or pay-forperformance, we immediately put our haunches up and try to work out how can we minimize any penalties to ourselves. But that's the wrong frameset for quality improvement. Quality improvement demands transparency not defensiveness. You want to be as open as you possibly can about where the problems might lie in your unit and what you can do about it. The strategy of changing your PEEP by 1 point per day clearly is not being done for any clinical benefit. The only reason to do that is purely to game the system. And you would only contemplate doing so, I think, if some sort of external impetus, such as public reporting or pay-for-performance, was driving you. What's disturbing about it is you're playing with the patient purely out of a desire to try to look good on some external metric.

Kacmarek: Let me add 2 other things to your list of causes that you talked about, but I don't think you made them explicit. One is not following mechanical ventilation guidelines and the other is physician bias in the application of mechanical ventilation. In your specific example, where you went from a PEEP of 5 and an $\mathrm{F}_{\mathrm{IO}_{2}}$ of 0.6 to a PEEP of 8 and an $\mathrm{F}_{\mathrm{IO}_{2}}$ of 0.4 could be a result of my bias for low PEEP and high $\mathrm{F}_{\mathrm{IO}_{2}}$, which is followed by you coming onto service and your bias for high PEEP and low $\mathrm{F}_{\mathrm{IO}_{2}}$. To what Lorenzo [Berra] said, all 14 of those obese subjects we studied $^{4}$ ended up with VAEs. They were all ventilated for a few days, they were all relatively stable, but the application of mechanical ventilation was not appropriate for their particular presentation. So when we righted the ship, they ended up with a VAE. Bill [Hurford], I think the most important thing about this is that it's an indicator of the global quality of the provision of mechanical ventilation, whether that be a result of lack of attention to detail by the various staff in the ICU, or not following guidelines, or biases, or just patient populations who get worse even if we do the right thing. But to your comment, the most important thing we should be doing is we should be drilling down on each and every VAE and determining the specific reason for that VAE. That's where I think the improvement in the way we operate will come.

Klompas: I completely agree. Your VAE paradox - this notion that improving the ventilation for obese patients can lead to a VAE-if I'm hearing you right, your conclusion is that your people weren't doing the right thing to begin with? So, that's a lesson learned, right? That when you put an obese patient on a ventilator, you need to speak to Bob [Kacmarek] or Lorenzo to work out how to do it right. That's a lesson that came out of VAE surveillance.

Kacmarek: Exactly. And if that's followed and drilled down, those examples will hopefully change our practice.

MacIntyre: One of the things I've always been bothered by is that PEEP and $\mathrm{F}_{\mathrm{IO}_{2}}$ are surrogate markers. For example, when patients are deteriorating and they go on airway pressure release ventilation or ECMO [extracorporeal membrane oxygenation], the applied PEEP may drop dramatically. These are patients who are clearly getting worse, and yet these indicators are going in the opposite direction. I'm concerned and I sort of favor Bill's idea of more direct measurements of care like duration of mechanical ventilation as a true indicator as to how you're doing on ventilator management. These more distant surrogate markers always scare me because there may be some unintended results from them, and they can be misleading.

Klompas: I have a question for you then, do you think a system built on mean airway pressures would be better?
MacIntyre: I still think these are surrogate markers that are chosen because they're easy to get off the computer record. That always worries me. I've been on committees where they say, "Well, we can't really measure the outcome, but we can measure this other number that we hope is a reasonable surrogate for this outcome." I have trouble with that. I'm a bottom line guy; if you're doing the right thing with the ventilator, your duration of mechanical ventilation will come down.

Kacmarek: Maybe, but maybe not, Neil. It depends on the particular patient you're dealing with. If you simply look at those outliers that have long stay and you don't have any criteria to guide your case, the amount of time you'd be spending on the chart to determine what potentially caused that patient to be mechanically ventilated for 2 or 3 weeks is enormous. At least here you're given some indicator of quality. And we'd all agree that it's not the only indicator and there are other things we can utilize, but it is a reasonable indicator that's easy to track and easy to deal with.

Hurford: Bob, woah. Where is this an indicator of quality? It's an indicator of ventilator management-that has nothing to do with quality.

Kacmarek: It's an indicator of the quality of ventilator management.

Hurford: I'd ask you to prove that.

Kacmarek: He showed a couple of slides, $37 \%$ preventable.

Hurford: Right, but what was the difference in patient outcome? Was there $37 \%$ less mortality? Was there $37 \%$ shorter duration of mechanical ventilation? There's probably a correlation, but show how an institution doing this in a randomized fashion really improves. It's a surrogate marker, like Neil said. 
Klompas: In our Wake Up And Breathe study 5 the $37 \%$ decrease in VAEs was accompanied by a 2.4-d decrease in mean duration of mechanical ventilation, a 3-d decrease in ICU stay, and a 6-d decrease in hospital stay.

Hurford: I would argue that that was not caused by the measurement but more likely because of the processes that you put into place.

Klompas: The processes were inspired by the measurement. We had the measurement, namely VAE. We then asked, "Well what are we going to do about this?' Analysis of VAEs led us to decide we need to improve our sedation and extubation practices. The measurement came down, reflecting the performance improvement, with the benefit being affirmed by the improvements in patient-centered outcomes. Six fewer days in the hospital is pretty good.

Mireles-Cabodevila: The challenge for us-I work in a medical ICU-is that compared to you, my patients stay on the ventilator much longer. And if I go to the neurological ICU, it's even worse. In my hospital, your outcomes are compared to other units. The rate of VAEs in my unit are higher than VAEs in the surgical ICU. And then we look bad.

Klompas: That's inherently unfair. You should be compared to the medical ICU at my hospital, not the surgical ICU in your hospital, you're right.

Berra: But the denominator is all patients who stay on the ventilator $>2 \mathrm{~d}$, right? So the surgical ICU at my hos- pital has the highest rate of VAE because very few patients overall stay on the ventilator for longer periods. And they're the same patients who can eventually get a VAE.

Klompas: The denominator is all patients receiving mechanical ventilation; it doesn't matter the minimum duration of ventilation. Which is a problem because you're actually only eligible for VAEs if you make it to $4 \mathrm{~d}$ or beyond. Including all ventilator days in the denominator, not just those beyond 3-4 d, was a simplification that the $\mathrm{CDC}$ has thus far maintained.

Hess: Back to Neil's point, ECMO is excluded. So one way to game the system is to put all your patients on ECMO.

MacIntyre: That's the way many are going!

Klompas: It's true. Because there's no reasonable way to apply VAE criteria to high frequency oscillatory ventilation or ECMO, they are excluded from surveillance. Fortunately, at my hospital, it's still few and far between. So, are we worried there is going to be an epidemic of people being put on ECMO in order to prevent VAEs? We'll have really lost the plot at that point.

Hess: And for airway pressure release ventilation, you just look at $\mathrm{F}_{\mathrm{IO}_{2}}$ and not the low pressure level because there was all kinds of confusion about how to you identify PEEP and $\mathrm{P}_{\text {low }}$ and such things.
Klompas: Yes. One of the nice things about the system is that there is an emerging and increasing body of literature on VAE. The CDC is, I think, paying very close attention to it and is being quite responsive. Every year, we see refinements to the system. Recent examples include a refinement of the list of antibiotics that qualify for infection-related ventilator-associated complication and a new rule wherein any PEEP $<5$ is treated as if it were 5 because of some concerns around spontaneous breathing trials. So the CDC is being very responsive. I think if we come up with a good way to include airway pressure release ventilation in the system, they would listen.

\section{REFERENCES}

1. Magill SS, Klompas M, Balk R, Burns SM, Deutschman CS, Diekema D, et al. Developing a new, national approach to surveillance for ventilator-associated events. Crit Care Med 2013;41(11):2467-2475.

2. Klompas M, Khan Y, Kleinman K, Evans RS, Lloyd JF, Stevenson K, et al. Multicenter evaluation of a novel surveillance paradigm for complications of mechanical ventilation. PLoS One 2011;6(3):e18062.

3. Klompas M, Magill S, Robicsek A, Strymish JM, Kleinman K, Evans RS, et al. Objective surveillance definitions for ventilator-associated pneumonia. Crit Care Med 2012;40(12):3154-3161.

4. Pirrone M, Fisher D, Chipman D, Imber DA, Corona J, Mietto C, et al. Recruitment maneuvers and positive end-expiratory pressure titration in morbidly obese ICU patients. Crit Care Med 2016;44(2):300-307.

5. Klompas M, Anderson D, Trick W, Babcock $\mathrm{H}$, Kerlin MP, Li L, et al. The preventability of ventilator-associated events. The CDCPrevention Epicenters Wake Up and Breathe Collaborative. Am J Respir Crit Care Med 2015;191(3):292-301.

This article is approved for Continuing Respiratory Care Education credit. For information and to obtain your CRCE

(free to AARC members) visit

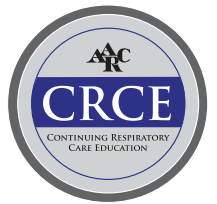

www.rcjournal.com 